\title{
Iteration functions for approximating complex roots of cubic polynomials
}

\author{
Enrico M. Yambao ${ }^{1 *} \mathcal{E}$ Ma. Carlota B. Decena ${ }^{1,2 *}$ \\ ${ }^{1}$ Department of Mathematics and Physics, College of Science; ${ }^{2}$ Research Center for the Natural \\ and Applied Sciences, University of Santo Tomas, 1015 Manila, Philippines
}

\begin{abstract}
Iterative methods provide an alternative to finding the solutions of equations where analytical methods are inconvenient or even impossible to use. This study which focuses on cubic polynomial equation $f(t)=a t^{3}+b t^{2}+c t+d=0, a>0$ with real coefficients and having an imaginary root, found that the fixed-point iteration $x_{n+1}=h\left(x_{n}\right)$ where $h(t)=1 / 3\left[f(t) / f^{\prime}(t)-\right.$ $b / a$ ] will always converge to the real part $x$ of the imaginary root of $f(t)=0$ whenever $b^{2}-$ $3 a c<0$. The only real root of $g(t)=1 / 2 f^{\prime}(t) f^{\prime \prime}(t)-a f(t)=0$ was found to be the real part $x$ of the imaginary root of $f(t)=0$ and is always outside the interval formed by the critical numbers of the function $f$.
\end{abstract}

Keywords: complex roots, iteration, cubic polynomial, zero of a function

\section{INTRODUCTION}

In numerical analysis, research on iterative approaches in finding the roots of different types of equations is highly relevant. It is mostly conducted and applied in the field of computer science [7, 9, 18-28]. Thus, most of the iterative approaches developed have been meant for encoding into computer algorithms, and not for solving by hand. This is particularly true when imaginary roots are desired. Such approaches employ a method that is complex, involving numerous tiers of computations to arrive at answers meeting the desired level of accuracy. Hence, their use in the typical numerical analysis lecture is highly limited. This limitation is also observed when students do not benefit much

*To whom correspondence should be addressed: mbdecena@ust.edu.ph / emyambao@ust.edu.ph from the current literature inasmuch as knowledge in programming languages is a prerequisite. This is the problem that this paper addresses.

Taking cue from the discovered neglect of current literature on iterative approaches that can be conducted by hand or basic computer software, previous studies revealed that the current methods are focused on determining the real roots of a polynomial equation. Likewise, it uses the approximation of the real root to reduce the equation further which subsequently allows the solution of the non-real roots to be found analytically. Other studies focused on imaginary roots but the iteration formulas involved are too complex or impractical to use when calculations are to be done by hand since computations are tedious when imaginary numbers are being used in the iteration process. Though it has been 
established in previous studies [3, 4, 7, 8, 13, 20, 26] that determining a real root becomes a prior step in finding non-real roots, there has yet to be an iterative approach that assumes to find the non-real roots without the aid of information coming from the estimated real roots. This is particularly true for cubic polynomials.

In this paper, an alternative iterative approach to finding the complex roots of cubic polynomial equations with real coefficients is presented. Apart from the existing methods, this new iterative approach finds the non-real roots independently of the approximated real root which guarantees greater accuracy.

We begin the approach with the assumption that imaginary roots of the cubic polynomial equation with real coefficients exist.

\section{THE ITERATION FUNCTIONS}

Fixed-point iteration. Consider the cubic polynomial equation with real coefficients

$$
f(t)=a t^{3}+b t^{2}+c t+d=0,(a>0)
$$

Assume that, $x+i y,(y \neq 0)$, is an imaginary root of (1). Then,

$$
\begin{gathered}
a(x+i y)^{3}+b(x+i y)^{2}+c(x+i y)+d=0 \\
{\left[\left(a x^{3}+b x^{2}+c x+d\right)-y^{2}(3 a x+b)\right]+} \\
y\left[\left(3 a x^{2}+2 b x+c\right)-a y^{2}\right] i=0 \\
{\left[f(x)-1 / 2 y^{2} f^{\prime \prime}(x)\right]+y\left[f^{\prime}(x)-a y^{2}\right] i=0}
\end{gathered}
$$

which holds only when $f(x)-1 / 2 y^{2} f^{\prime \prime}(x)=0$ and

$$
y\left[f^{\prime}(x)-a y^{2}\right]=0
$$

Since $y \neq 0$, then (2) holds only when $f^{\prime}(x)-a y^{2}$ $=0$ or when

$$
y^{2}=f^{\prime}(x) / a
$$

Substituting (3) into (2) yields

$$
f^{\prime \prime}(x) / 2 a=f(x) / f^{\prime}(x)
$$

Since $f^{\prime \prime}(x) / 2=3 a x+b$, then $x=1 / 3\left[f(x) / f^{\prime}(x)-\right.$ $b / a]$, provided $f^{\prime}(x) \neq 0$.
Thus, the real part of the imaginary root of (1) is the fixed point of the function defined by

$$
h(t)=1 / 3\left[f(t) / f^{\prime}(t)-b / a\right]
$$

It is evident from (3) that at the fixed point $x$ of the function $h, f^{\prime}(x)>0$. Hence, the fixed point iteration for the real part of the imaginary root of (1) is

$$
\begin{aligned}
& x_{n+1}=h\left(x_{n}\right)=1 / 3\left[f\left(x_{n}\right) / f^{\prime}\left(x_{n}\right)-b / a\right], f^{\prime}\left(x_{n}\right) \neq 0, \\
& n=0,1,2, \ldots
\end{aligned}
$$

If this iteration converges, by virtue of (3), in any stage of the iteration process, the approximations to the imaginary parts of the root are

$$
y_{n}= \pm \sqrt{f^{\prime}\left(x_{n}\right) / a}
$$

If $r$ is the real root of (1), then $r+(x+i y)+(x-i y)$ $=r+2 x=-b / a$. Thus, in any stage of the iteration process, the approximation to the real root of (1) is

$$
r_{n}=-2 x_{n}-b / a
$$

Establishing the condition for convergence. The fixed-point iteration (6) converges if in the vicinity of the fixed point $x$ of $h,\left|h^{\prime}(t)\right|<1$, or without any loss in generality, if at the fixed point $x,\left|h^{\prime}(x)\right|<1$.

From (5), $h^{\prime}(t)=1 / 3\left[1-f(t) f^{\prime \prime}(t) /\left[f^{\prime}(t)\right]^{2}\right]$

At the fixed point $x$,

$$
\begin{aligned}
& f(x) / f^{\prime}(x)=f^{\prime \prime}(x) / 2 a \text { and } h^{\prime}(x) \\
& =1 / 3\left[1-f(x) f^{\prime \prime}(x) /\left[f^{\prime}(x)\right]^{2}\right] \\
& =-1 / 3\left[5+2\left(b^{2}-3 a c\right) / a f^{\prime}(x)\right]
\end{aligned}
$$

Thus,

$$
\left|h^{\prime}(x)\right|=1 / 3\left|5+2\left(b^{2}-3 a c\right) / a f^{\prime}(x)\right| .
$$

If $b^{2}-3 a c \geq 0$, since $a>0$ and $f^{\prime}(x)>0,\left|h^{\prime}(x)\right|$ $=1 / 3\left|5+2\left(b^{2}-3 a c\right) / a f^{\prime}(x)\right| \geq 5 / 3>1$.

Hence, the iteration (6) does not converge in this case. However, if $b^{2}-3 a c<0$, then $-2\left(b^{2}-\right.$ $3 a c)>0$. 
Since $f^{\prime}(x) \geq-b^{2}-3 a c / 3 a>0$,

$$
\begin{gathered}
1 / f^{\prime}(x) \leq-3 a / b^{2}-3 a c \\
1 / a f^{\prime}(x)<-3 / b^{2}-3 a c \\
-2\left(b^{2}-3 a c\right) / a f^{\prime}(x)<-3\left[-2\left(b^{2}-3 a c\right)\right] / b^{2}-3 a c \\
1 / 3\left[-5-\left(b^{2}-3 a c\right) / a f^{\prime}(x)\right]<1 / 3
\end{gathered}
$$

Consequently, $h^{\prime}(x)=1 / 3\left[-5-2\left(b^{2}-3 a c\right) / a f^{\prime}(x)\right]$ $<1 / 3$ and the fixed point iteration (6) converges since $\left|h^{\prime}(x)\right|<1$.

This result is stated in the following theorem:

\section{Theorem: The fixed point iteration}

$$
x_{n+1}=h\left(x_{n}\right)=1 / 3\left[f\left(x_{n}\right) / f^{\prime}\left(x_{n}\right)-b / a\right], f^{\prime}\left(x_{n}\right) \neq 0
$$

will converge to the real part $x$ of the imaginary root of the cubic polynomial equation $f(t)=$ $a t^{3}+b t^{2}+c t+d=0$ whenever $b^{2}-3 a c<0$. In addition, in any stage of the iteration process, the approximations to the real root $r$ and the imaginary part $y$ of the imaginary root are given, respectively, by

$$
r_{n}=-2 x_{n}-b / a \text { and } y_{n}= \pm \sqrt{f^{\prime}\left(x_{n}\right) / a} .
$$

In the previous theorem, the convergence of the identified fixed-point iteration in finding the complex roots is established based on the condition $b^{2}-3 a c<0$. In the case when $b^{2}-3 a c$ $\geq 0$, convergence is guaranteed for another iteration function which is shown next.

Newton-Raphson iteration. From (4), we have $1 / 2 f^{\prime}(x) f^{\prime \prime}(x)-a f(x)=0$. Define $g(t)=1 / 2$ $f^{\prime}(t) f^{\prime \prime}(t)-a f(t)$. Clearly, the real part $x$ of the imaginary root of $f(t)=0$ is a real root of the equation $g(t)=0$. Now, $g(t)=1 / 2 f^{\prime}(t) f^{\prime \prime}(t)-$ $a f(t)=1 / 2\left(3 a t^{2}+2 b t+c\right)(6 a t+2 b)-a\left(a t^{3}+b t^{2}+\right.$ $c t+d)$. Thus, $g(t)=8 a^{2} t^{3}+8 a b t^{2}+2\left(a c+b^{2}\right) t+$ $(b c-a d)$.

Let $A=8 a^{2}, B=8 a b, C=2\left(a c+b^{2}\right)$, and $D=b c-$ $a d$. If $b^{2}-3 a c \geq 0$, then

$$
\begin{gathered}
B^{2}-3 A C=(8 a b)^{2}-3\left(8 a^{2}\right)\left[2\left(a c+b^{2}\right)\right]=16 a^{2}\left(b^{2}\right. \\
-3 a c) \geq 0
\end{gathered}
$$

Thus, $\left(B^{2}-3 A C\right)^{3 / 2}=64 a^{3}\left(b^{2}-3 a c\right)^{3 / 2}$.

Similarly,

$$
\begin{gathered}
27 A^{2} D+2 B^{3}-9 A B C=27\left(8 a^{2}\right)^{2}(b c-a d)+ \\
2(8 a b)^{3}-9\left(8 a^{2}\right)(8 a b)\left[2\left(a c+b^{2}\right)\right] \\
=-64 a^{3}\left(27 a^{2} d+2 b^{3}-9 a b c\right)
\end{gathered}
$$

Since $a>0, b^{2}-3 a c \geq 0$, and $\mid 27 a^{2} d+2 b^{3}-$ $9 a b c \mid>2\left(b^{2}-3 a c\right)^{3 / 2}$, then

$$
\begin{aligned}
\mid 27 A^{2} D+ & 2 B^{3}-9 A B C\left|=64 a^{3}\right| 27 a^{2} d+ \\
& 2 b^{3}-9 a b c \mid \\
> & 64 a^{3}\left[2\left(b^{2}-3 a c\right)^{3 / 2}\right] \\
= & 2\left[64 a^{3}\left(b^{2}-3 a c\right)^{3 / 2}\right] \\
= & 2\left(B^{2}-3 A C\right)^{3 / 2}
\end{aligned}
$$

Consequently, the coefficients of $g(t)$ satisfy (2). Hence, $g(t)=0$ has an imaginary root and its only real root is the real part of the imaginary root of $f(t)=0$. From $g(t)=1 / 2 f^{\prime}(t) f^{\prime \prime}(t)-a f(t)$,

$$
\begin{gathered}
g^{\prime}(t)=1 / 2\left[f^{\prime \prime}(t) f^{\prime \prime}(t)+f^{\prime}(t) f^{\prime \prime \prime}(t)\right]-a f^{\prime}(t) \\
=1 / 2\left[f^{\prime \prime}(t)\right]^{2}+2 a f^{\prime}(t)
\end{gathered}
$$

Newton-Raphson iteration for the real root $x$ of $g(t)=0$ is $x_{n+1}=x_{n}-g\left(x_{n}\right) / g^{\prime}\left(x_{n}\right), g^{\prime}\left(x_{n}\right) \neq 0$, $n=0,1,2, \ldots$ or equivalently,

$$
x_{n+1}=x_{n}-\frac{1 / 2 f^{\prime}\left(x_{n}\right) f^{\prime \prime}\left(x_{n}\right)-a f\left(x_{n}\right)}{1 / 2\left[f^{\prime \prime}\left(x_{n}\right)\right]^{2}+2 a f^{\prime}\left(x_{n}\right)},
$$

provided,

$$
1 / 2\left[f^{\prime \prime}\left(x_{n}\right)\right]^{2}+2 a f^{\prime}\left(x_{n}\right) \neq 0
$$

The approximations to the imaginary parts and real root of $f(t)=0$ corresponding to the iterates generated by this iteration can be obtained using (7) and (8), respectively.

\section{Establishing the interval that contains the real} part of the imaginary root. In any typical iteration procedure, the efficiency of an iteration depends not only on the iteration function but also on the starting approximation $x_{0}$. It must be taken from an interval that contains the desired root. The interval containing the real part of an imaginary root can be established based on the graphs of $f$ and the function $g$ used in Newton- 
Raphson iteration. Being a cubic polynomial, some properties of $g$ can be obtained from the properties of $f$. Since $g(t)=A t^{3}+B t^{2}+C t+D$, where $A=8 a^{2}, B=8 a b, C=2\left(a c+b^{2}\right)$, and $D=b c$ - $a d$, the local extrema of $g$, if there are any, occur at

$t=\frac{-B \pm \sqrt{B^{2}-3 A C}}{3 A}=\frac{-2 b \pm \sqrt{b^{2}-3 a c}}{6 a}=-\frac{b}{3 a} \pm \frac{\sqrt{b^{2}-3 a c}}{6 a}$

and its point of inflection occurs at

$$
t=-\frac{B}{3 A}=-\frac{b}{3 a} .
$$

Consequently, the critical numbers of $g$ are always within the interval formed by the critical numbers of $f$ and their points of inflection occur at the same number $t=-b / 3 a$. At their points of inflection,

$$
\begin{aligned}
f\left(-\frac{b}{3 a}\right)= & a\left(-\frac{b}{3 a}\right)^{3}+b\left(-\frac{b}{3 a}\right)^{2}+c\left(-\frac{b}{3 a}\right)+d \\
& =\frac{27 a^{2} d+2 b^{3}-9 a b c}{27 a^{2}}
\end{aligned}
$$

and

$$
g-\frac{b}{3 a}=g-\frac{B}{3 A}=\begin{gathered}
27 A^{2} D+2 B^{3}-9 A B C \\
27 A^{2}
\end{gathered}
$$

or equivalently,

$$
g\left(-\frac{b}{3 a}\right)=-a \frac{27 a^{2} d+2 b^{3}-9 a b c}{27 a^{2}}=-a f\left(-\frac{b}{3 a}\right)
$$

Note the local extrema of $f$, if there are any, are

$$
\begin{gathered}
f\left(\frac{-b \pm \sqrt{b^{2}-3 a c}}{3 a}\right) \\
=\frac{27 a^{2} d+2 b^{3}-9 a b c+2\left(b^{2}-3 a c\right)^{3 / 2}}{27 a^{2}} .
\end{gathered}
$$

The local extrema of are,

$$
\begin{gathered}
g\left(-\frac{b}{3 a} \pm \frac{\sqrt{b^{2}-3 a c}}{6 a}\right) \\
=-a\left(\frac{27 a^{2} d+2 b^{3}-9 a b c \pm 2\left(b^{2}-3 a c\right)^{3 / 2}}{27 a^{2}}\right) \\
=a f\left(\frac{-b \mp \sqrt{b^{2}-3 a c}}{3 a}\right)
\end{gathered}
$$

Clearly, the corresponding local extrema and points of inflection of the graphs of $f$ and $g$ lie on the opposite sides of the horizontal axis. From the preceding section,

$$
\begin{aligned}
B^{2}-3 A C & =(8 a b)^{2}-3\left(8 a^{2}\right)\left[2\left(a c+b^{2}\right)\right] \\
& =16 a^{2}\left(b^{2}-3 a c\right)
\end{aligned}
$$

Hence, if $f$ is a monotone, then so is $g$ and if $f$ is not, then so is $g$. In case $f$ is a monotone, that is, when $b^{2}-3 a c<0$, the only real root $x$ of $g(t)=0$ must be greater than $-b / 3 a$ if the point of inflection of $f$ is above the horizontal axis. This root is less than $-b / 3 a$ if the point of inflection is below the horizontal axis. When the point of inflection is on the horizontal axis, then $x=-b$ / $3 a$ provided $f^{\prime}(-b / 3 a) \neq 0$. Based on Fig. below, in case $f$ is not a monotone, that is, when $b^{2}-$ $3 a c>0$, the point of inflection and local extrema of $f$ are on the same side of the horizontal axis.

On the interval formed by the critical numbers of $f, f^{\prime}(t)<0$. The real part $x$ of the imaginary root of $f(t)=0$ cannot be contained in this interval since from the previous part of this chapter, it is necessary that $f^{\prime}(x)>0$. Thus, the graph of $g$ must cross the horizontal axis at a point to the right of the local minimum point of $f$ if the point of inflection of $f$ is above the horizontal axis. Similarly, the graph of $g$ must

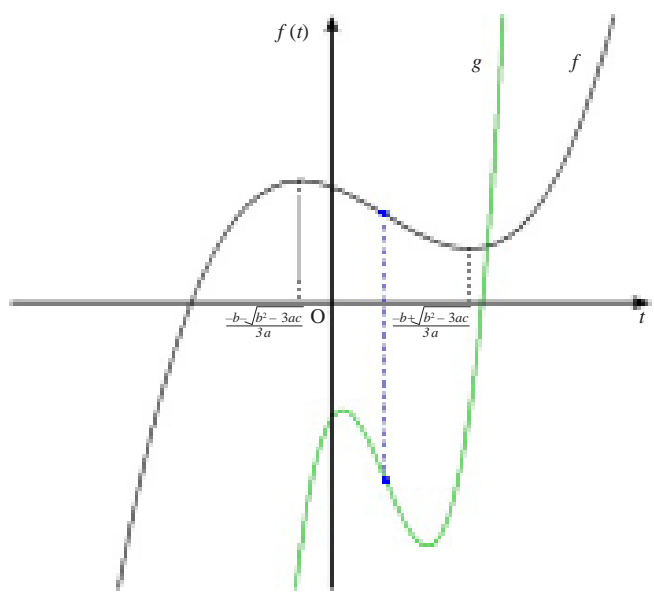

Graphs of $f$ and $g$ having point of inflection and local extrema on one side of the $\mathrm{x}$-axis 
cross the horizontal axis at a point to the left of the local maximum point of $f$ if the point of inflection of $f$ is below the horizontal axis.

This generalization is stated in Theorem 2 .

\section{Theorem 2}

Let $f(t)=a t^{3}+b t^{2}+c t+d=0,(a>0)$, be a cubic polynomial equation that has an imaginary root $x+i y$.

i. If $b^{2}-3 a c<0$, then $x \geq-b / 3 a$ whenever $f(-\mathrm{b} /$ $3 a) \geq 0$ and $x<-b / 3 a$ whenever $f(-b / 3 a)<0$.

ii. If $b^{2}-3 a c>0$, then

$$
\begin{gathered}
x>\frac{-b+\sqrt{b^{2}-3 a c}}{3 a} \text { whenever } f\left(-\frac{b}{3 a}\right)>0 \\
\text { and } x<\frac{-b-\sqrt{b^{2}-3 a c}}{3 a} \text { whenever } f\left(-\frac{b}{3 a}\right)<0 .
\end{gathered}
$$

\section{CONCLUSION}

The difficulty in using the fixed-point iteration in solving any equation is in finding the equivalent equation to identify the iteration function that will ensure that the iteration will converge to the desired root. This is usually being accomplished by trial and error. The fixed point-iteration $x_{n+1}=1 / 3\left[f\left(x_{n}\right) / f^{\prime}\left(x_{n}\right)-b / a\right]$ for the real part of the imaginary root of the cubic polynomial equation $f(t)=a t^{3}+b t^{2}+c t+d=0$ offers an alternative to find or generate all the possible roots of this equation without trial and error provided $b^{2}-3 a c<0$. The NewtonRaphson iteration is known to be a powerful tool in finding the zero of a function especially if the root is contained in an interval where the function is increasing or decreasing. Thus, in case $b^{2}-3 a c \geq 0$ and $\left|27 a^{2} d+2 b^{3}-9 a b c\right|>$ $2\left(b^{2}-3 a c\right)^{3 / 2}$, the Newton-Raphson iteration $x_{n+1}$ $=x_{n}-g\left(x_{n}\right) / g^{\prime}\left(x_{n}\right)$, where $g(t)=1 / 2 f^{\prime}(t) f^{\prime \prime}(t)-$ af $(t)$, is expected to converge to the real part of the imaginary root of $f(t)=0$ provided the initial approximation $x_{0}$ is properly chosen in the interval $\left(-\infty, \frac{-b-\sqrt{b^{2}-3 a c}}{3 a}\right) \cup\left(\frac{-b+\sqrt{b^{2}-3 a c}}{3 a},+\infty\right)$.

This is the interval that contains the real part $x$ and $f$ is strictly increasing (or decreasing when $a<0$ ) over this interval.

\section{FUTURE OUTLOOK}

Future investigation maybe undertaken on the functions used in this approach as to determine how it can be modified and extended to cubic polynomial equations with complex coefficients in general. Since the function $g$ used in NewtonRaphson iteration preserves the orientation of the graph and the nature of the zeros of the cubic polynomial $f$, it is also recommended that its other properties be further explored particularly in complex setting.

\section{REFERENCES}

[1] Argyros IK, Hilout S. Extending the Newton Kantorovich hypothesis for solving equations. Journal of Computational and Applied Mathematics 2010; 234:2993-3006.

[2] Carstensen C, Petkovic M. On iteration methods without derivatives for the simultaneous determination of polynomial zeros. Journal of Computational and Applied Mathematics 1993; 45:251-66.

[3] Drakopoulos V. On the additional fixed points of Schroder iteration functions associated with a one iteration family of cubic polynomials. Computation and Graphics 1998; 22(5):639-34.

[4] Fang T, Fang G, Lee C. A new iteration method with cubic convergence to solve nonlinear algebraic equations. Applied Mathematics and Computation 2006; 175(2):1147-55.

[5] Gutierrez JM, Hernandez MA, Romero N. Dynamics of a new family of iterative processes for quadratic polynomials. Journal of Computational and Applied Mathematics 2010; 233:2688-95.

[6] Herceg D, Petkovic L, Petkovic MS. On a simultaneous method of Newton-Weierstrass type for finding all zeros of a polynomial. Applied Mathematics and Computation 2009; 215:245663. 
[7] Homeier HHH. A modified Newton method for rootfinding with cubic convergence. Journal of Computational and Applied Mathematics 2003; 157:227-30.

[8] Ivanisov AV, Polishchuck VK. A method of finding the roots of polynomials which converges for initial approximation. Computational Mathematics on Mathematical Physics 1985; 25(3):1-7.

[9] Koçak M. Ostrowski's fourth-order iterative method speedily solves cubic equations of state. Journal of Computational and Applied Mathematics 2010. DOI:10.1016/ j.cam.2010.09.012

[10] Lin B, Liu L, Wang G, Zhang L. Fast approach for computing roots of polynomials using cubic clipping. Computer Aided Design 2009; 26:54759.

[11] Lu L. Sample-based polynomial approximation of rational Bézier curves. Journal of Computational and Applied Mathematics 2011; 235:1557-63.

[12] Mclaughlin JB. Convergence of a relaxed Newton's method for cubic equations. Computers Chemical Engineering 1993; 17(10):971-83.

[13] Nikkah-Bahrami M, Oftadeh R. An effective iterative method for computing real and complex roots of systems of nonlinear equations. Applied Mathematics and Computation 2009; 215:181320.

[14] Nourein AM. An iteration formula for the simultaneous determination of the zeros of a polynomial. Journal of Computational and Applied Mathematics 1975; 1(4):251-54.

[15] Pakdemirli M, Yurtsever HA. Estimating roots of polynomials using perturbation theory. Applied Mathematics and Computation 2007; 188:20252008?.

[16] Pan VY, Zheng A. New progress in real and complex polynomial root-finding. Computers and Mathematics with Applications 2011; 61:130534.

[17] Parida P, Gupta D. A cubic convergent iterative method for enclosing simple roots of nonlinear equations. Applied Mathematics and Computation 2007; 187(2):1544-51.

[18] Petkovic LD, Petkovic MS. Construction of zerofinding methods for Weierstrass functions. Applied Mathematics and Computation 2007; 184:351-59.
[19] Petkoviæ MS. The self-validated method for polynomial zeros of high efficiency. Journal of Computational and Applied Mathematics 2009; 233(4):1175-86.

[20] Petkovic MS. A highly efficient root-solver of very fast convergence. Applied Mathematics and Computation 2008; 205:298-302.

[21] Petkovic MS. Comments on some recent methods for the simultaneous determination of polynomial zeros. Journal of Computational and Applied Mathematics 2002; 145:519-24.

[22] Petkovic MS. Some interval iterations for finding a zero of a polynomial with error bounds. Computers and Mathematics with Applications 1987; 14(6):479-95.

[23] Petkovic M, Milovanovic G, Stefanovic L. Some higher-order methods for the simultaneous approximation of multiple polynomial zeros. Computers \& Mathematics with Applications 1986; 12(9):951-62.

[24] Petkovic MS, Milovanovic GV. A note on some improvements of the simultaneous methods for determination of polynomial zeros. Journal of Computational and Applied Mathematics 1983; 9:65-69.

[25] Petkovic MS, Petkovic L, Rancic LZ. Point estimation of simultaneous methods for solving polynomial equations: A survey (II). Journal of Computational and Applied Mathematics 2007; 205:32-52.

[26] Petkovic MS, Rancic L. A new fourth-order family of simultaneous methods for finding polynomial zeros. Applied Mathematics and Computations 2005; 164:227-48.

[27] Petkovic MS, Stefanovic LV. On some improvements square root iteration for polynomial complex zeros. Journal of Computational and Applied Mathematics 1986; 15:13-25.

[28] Petkovic M, Trickovic S. A Family of Simultaneous Zero-Finding Methods.Computers Math Applic 1997; 34(10):49-59.

[29] Sweilam NH. Variational iteration method for solving cubic nonlinear Schrodinger equation. Journal of Computational and Applied Mathematics 1997; 207:155-63.

[30] Strobach P. Solving cubics by polynomial fitting. Journal of Computational and Applied Mathematics 2011; 235:3033-52.

Graphs of $f$ and $g$ having point of inflection and local extrema on one side of the $x$-axis 